\title{
Multidimensional Radar Waveforms \\ A New Paradigm for the Design and Operation of Highly Performant Spaceborne Synthetic Aperture Radar Systems
}

\author{
Gerhard Krieger, Nicolas Gebert, Alberto Moreira \\ Microwaves and Radar Institute \\ German Aerospace Center (DLR) \\ Oberpfaffenhofen, Germany \\ E-Mail: gerhard.krieger@dlr.de
}

\begin{abstract}
This paper introduces and analyses the innovative paradigm of multidimensional waveform encoding for spaceborne synthetic aperture radar (SAR). The combination of this technique with digital beamforming on receive enables a new class of highly performant SAR systems employing novel and highly flexible radar imaging modes. Examples are adaptive high-resolution wide-swath SAR imaging with compact antennas, enhanced parameter estimation sensitivity for applications like along-track interferometry and moving object indication, and the implementation of hybrid SAR imaging modes that are well suited to satisfy the hitherto incompatible user requirements for frequent monitoring and detailed mapping. Implementation specific issues will be discussed and examples demonstrate the potential of the new technique for different remote sensing applications.
\end{abstract}

\section{INTRODUCTION}

The unambiguous swath width and the achievable azimuth resolution pose contradicting requirements on the design of spaceborne synthetic aperture radar (SAR) systems [1]. This motivated the development of advanced SAR imaging modes with different trade-offs between spatial coverage and azimuth resolution. Examples are the ScanSAR mode which enables a wide imaging swath at the cost of an impaired azimuth resolution [2] and the Spotlight mode which allows for an improved azimuth resolution on the cost of a noncontiguous imaging along the satellite track [3]. It is, however, not possible to combine both imaging modes simultaneously in one and the same data take. This dilemma motivated further research towards the development of new radar techniques for spaceborne high-resolution wide-swath SAR imaging.

A promising candidate for such a new radar imaging technique is digital beamforming on receive where the receiving antenna is split into multiple sub-apertures (cf. Fig. 1). In contrast to analog beamforming, the received signals from each sub-aperture element are separately amplified, down-converted, and digitized. This enables an a posteriori combination of the recorded sub-aperture signals to form multiple beams with adaptive shapes. The additional information about the direction of the scattered radar echoes can then be used to (1) suppress spatially ambiguous signal returns from the ground, (2) to increase the receiving antenna gain without a reduction of the imaged

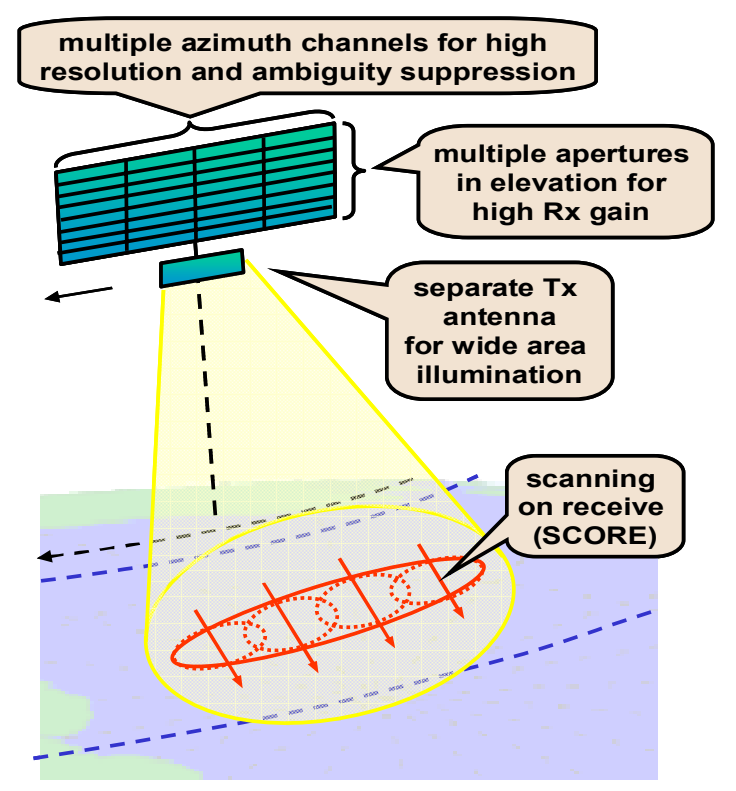

Figure 1. "Classical” High-Resolution Wide-Swath SAR System.

area, (3) to suppress spatially localized interferences, and (4) to gain additional information about the dynamic behavior of the scatterers and their surroundings. By this, it becomes possible to overcome the fundamental limitations of conventional SAR systems [4]-[10].

A prominent example for the recent developments is the high-resolution wide-swath (HRWS) SAR system which combines a small transmit antenna with a large receiver array as illustrated in Fig. 1 [6][8]. The small transmit antenna illuminates a wide swath on the ground and the large receiver array compensates the Tx gain loss by a real time digital beamforming process called scanning on receive (SCORE). Multiple azimuth channels allow furthermore for the acquisition of additional samples along the synthetic aperture by employing the principle of the displaced phase centre antenna (DPCA, [4]). This enables a reduction of the pulse repetition frequency (PRF) and therefore the imaging of a wider swath without rising azimuth ambiguities. The combination of the azimuth signals from the multiple apertures requires the application of dedicated multi-channel SAR signal processing algorithms as introduced in [7] and further elaborated in [9]. 


\section{Multidimensional WAVEFORM ENCODING}

The HRWS system concept assumes a wide area illumination by a separate transmit antenna. This enables an independent electrical design and optimization of the transmit and receive paths, but it requires also the accommodation of an additional antenna on the spacecraft and reduces the flexibility to operate the radar system in different SAR imaging modes like ultra-wide-swath ScanSAR, high SNR spotlight, or new hybrid modes to be discussed later. It is hence worth to consider also the application of digital beamforming techniques in radar systems that use the same antenna array for both the transmission and reception of radar pulses, thereby taking advantage of already existing space-qualified $\mathrm{T} / \mathrm{R}$ module technology. Since the high-resolution wide-swath SAR imaging capability is essentially based on a large antenna array, this poses in turn the question of how to distribute the signal energy on the ground. The trivial solution would be amplitude tapering, or as an extreme case, the use of only a part of the antenna for signal transmission, but this causes a significant loss of efficiency. Another possible solution is phase tapering, but the derivation of appropriate phase coefficients is an intricate task which requires in general complicated numerical optimization techniques.

A different and completely novel approach to exploit the large SAR antenna array is the use of spatiotemporally non-separable waveforms for each transmitted radar pulse. Such waveforms are characterized by the inequality

$$
w\left(t, \theta_{e l}, \theta_{a z}\right) \neq h(t) \cdot a\left(\theta_{e l}\right) \cdot b\left(\theta_{a z}\right)
$$

where $h(t)$ describes the temporal modulation of the transmitted radar pulse, $a\left(\theta_{e l}\right)$ the weighting from the antenna pattern in elevation, and $b\left(\theta_{a z}\right)$ the weighting from the antenna pattern in azimuth. The illustration in Fig. 2 visualizes the difference between a non-separable waveform encoding (right) and a separable transmit pulse (left) as used in all conventional SAR imaging modes and systems.
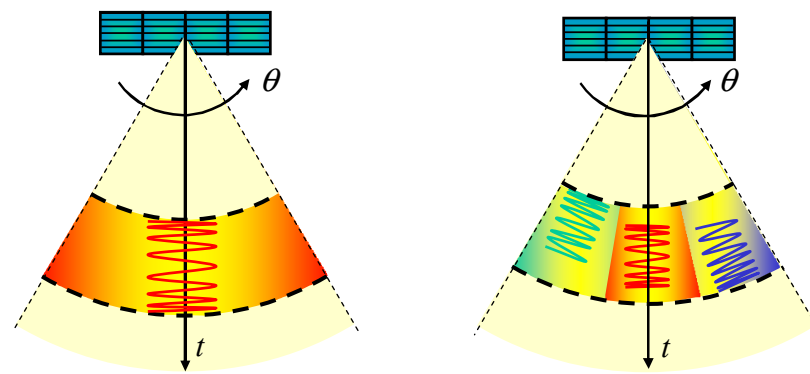

Figure 2. Separable and non-separable Tx waveforms. Left: Separable radar pulse as used in all conventional SAR systems and imaging modes. Right: Non-separable waveform allowing for a multidimensional encoding of the transmitted radar pulse.

A simple example for a non-separable waveform encoding in space and time is a mere switching between different antenna beams and/or sub-aperture elements during each transmitted pulse. The overall PRF remains unaltered in this case. Full range resolution within each sub-beam is achieved by concatenating multiple chirp signals in a sawtooth like frequency modulation (or any other sequence of full bandwidth and possibly even mutually orthogonal waveforms). The scheme allows a staggered illumination of a large area during each pulse, thereby supporting a systematic distribution of the available signal energy within this area. The opportunity for wide swath illumination with multiple sub-pulses will be investigated in more detail in Sect. III. A further advantage arises for improved azimuth ambiguity suppression by employing a reduced antenna beamwidth for each sub-pulse. This will be discussed together with other advantages in Sect. IV.

The concept of multidimensional waveform encoding can of course be extended to an arbitrary spatiotemporal radar illumination where each direction has its own temporal transmit signal with different power, duration, and/or phase code. Still another opportunity is a systematic decomposition of the overall transmitted range frequency spectrum into multiple sub-bands. Each sub-band is then associated with a different sub-aperture of the antenna array. Such a frequency decomposition of the transmitted range pulse may also be combined with intra-pulse aperture switching and/or beam steering in azimuth as introduced in Sect. IV. By this, it becomes possible to illuminate a large footprint on the ground notwithstanding the extended size of the total Tx antenna array in elevation and to simultaneously improve the suppression of azimuth ambiguities for a given antenna length.

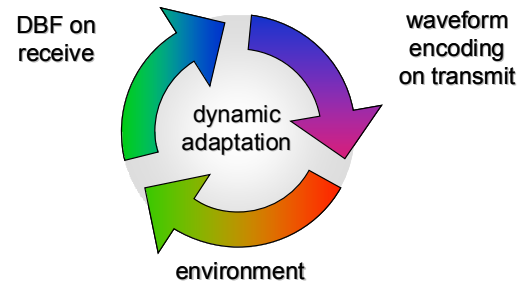

Figure 3. Dynamic adaptation of the waveform encoding to the environment by closing the loop between receiver and transmitter.

The selection of the spatiotemporal excitation coefficients for the individual Tx apertures could even be made adaptive by evaluating the recorded samples from previous signal returns (cf. Fig. 3). By this, a closed loop will be formed between the radar sensor and its environment, which allows for a maximization of the information that can be derived about the imaged scene for a given RF power budget. In analogy to the information theoretic modeling of multiple-input multiple-output (MIMO) communication systems, such an optimization could then be regarded as maximizing the mutual information between the recorded radar signals and the scatterer distribution on the ground, thereby making optimum use of the channel capacity provided by the multiple antenna $\mathrm{Tx} / \mathrm{Rx}$ radar system. For illustration, one may consider the simple case of an automatic compensation of angular variations in the received $\mathrm{Rx}$ power being caused by, e.g., range differences, inhomogeneous atmospheric RF signal attenuation, and/or spatial variations in the first-order scattering statistics of the imaged scene.

The full exploitation of all opportunities arising from such an adaptive multidimensional waveform encoding requires of course new SAR system design and optimization strategies. For example, the derivation of optimized waveforms may incorporate elements from Shannon's information theory. This will not only help to maximize the information content derived from the imaged scene, but it is also well suited to get rid of unnecessary redundancies in the recorded data from a multi-aperture SAR system [10]. 


\section{INTRA-PUlse BEAMSTEERING IN ELEVATION}

One example for multidimensional waveform encoding is intra-pulse beam steering in elevation. This enables an illumination of a wide image swath with a sequence of narrow and high gain antenna beams. Such a staggered illumination is in some sense similar to the traditional ScanSAR mode, with the important difference that each transmitted pulse illuminates now not only one but all subswaths simultaneously. The illumination sequence within each Tx pulse can in principle be arranged in any order. An interesting opportunity arises if we start from far range illumination and proceed consecutively to near range as illustrated in Fig. 4. As a result, the radar echoes from different sub-swaths will overlap in the receiver as shown in Fig. 4 on the upper right. The overall receiving window can hence be shortened, thereby reducing the amount of data to be recorded and stored on the satellite without the necessity for real-time on-board processing as in the SCORE process of the HRWS system. The temporal overlap of the radar echoes from the different sub-swaths is then resolved in the spatial domain by digital beamforming on receive. This a posteriori processing can be performed off-line on the ground, which has the further advantage that no information about the spatial structure of the recorded radar data will be lost, thereby enabling e.g. a suppression of directional interferences or jamming signals and avoiding the mountain clipping problem of the realtime SCORE technique as discussed in [10].

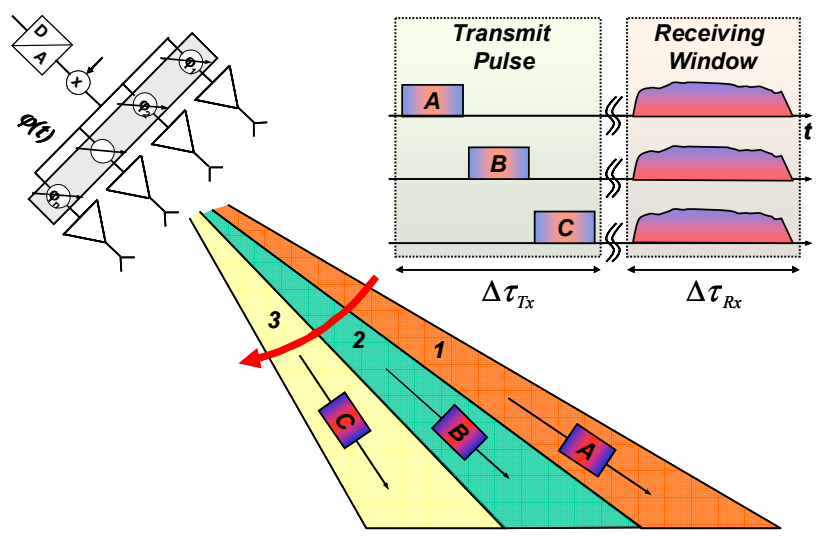

Figure 4. Intra-pulse beamsteering in elevation: The backscattered signals from different sub-swaths superimpose in the receiving window.

A direct consequence of the shortened receiving window length is the increased time to transmit multiple subpulses. This reduces the RF peak power requirements in the transmitter and provides further margin to switch between the sub-pulses, thereby simplifying the electrical system design. Another advantage of the staggered illumination is the reduced gain loss at the border of the swath if compared to a conventional radar illuminator. The use of variable Tx sub-pulses allows even for a flexible distribution of the signal energy on the ground. As a simple example one may consider the use of longer transmit pulses for sub-beams with higher incident angles. This illumination strategy is well suited to compensate the SNR loss due to both the typical decrease of the backscattering with increasing incident angles and the additional free space loss from a larger range. As a result, one may reduce the overall power requirements of the radar payload which in turn alleviates the thermal and electrical design of the satellite.

\section{WAVEFORM ENCODING IN AZIMUTH}

Waveform diversity in the radar transmitter can increase the information about the direction of a given scatterer. A simple example is a multi-aperture antenna where each aperture transmits its own orthogonal waveform. The orthogonality enables a separation of the radar echoes from the different transmit signals and the spatial diversity of the transmit phase centers causes relative phase shifts between the received waveforms for a given scatterer on the ground. This additional information can then be used to suppress ambiguous returns from point like targets or to increase the sensitivity to object movements by evaluating systematic phase differences between the orthogonal radar echoes.
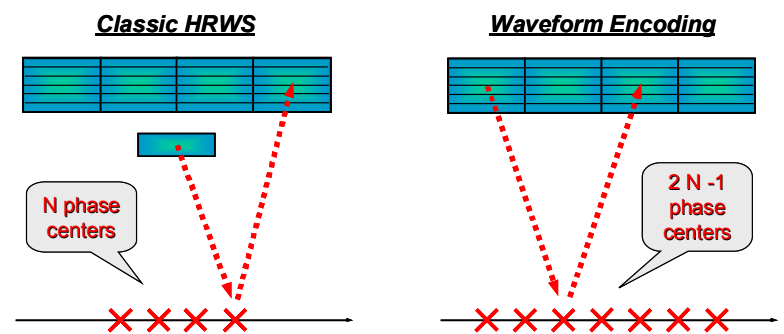

Figure 5. Separable and non-separable waveforms. Left: Separable radar pulse as used in all conventional SAR systems and imaging modes. Right: Non-separable waveform allowing for a multidimensional encoding of the transmitted radar pulse.

The performance gain from multidimensional waveform encoding can also be understood by considering the additional effective phase centre positions resulting from a multi-aperture Tx/Rx system. Fig. 5 compares the effective phase centre positions of the HRWS system (left) with the multidimensional waveform encoding technique (right). For the HRWS system, which combines a single fixed illuminator with a multi-channel receiver, one obtains for each transmitted pulse in total $N_{R x}$ effective phase centers. Their positions are spatially separated by a distance of $d_{\text {ant }} / 2$ where $d_{\text {ant }}$ is the distance between the Rx apertures in the along-track direction. The maximum distance of the phase centers is then given by

$$
d_{\max }=\frac{N_{R x}-1}{2} \cdot d_{\text {ant }}
$$

where $N_{R x}$ is the number of channels in azimuth. The use of multidimensional waveform encoding leads now to additional phase centers, since we have to consider each $\mathrm{Tx} / \mathrm{Rx}$ aperture pair (cf. Fig. 5, right). If we assume the same number $N=N_{R x}=N_{T x}$ and equal positions for the Tx and $\mathrm{Rx}$ apertures, we obtain in total $2 N-1$ independent phase centre positions which span a total length of

$$
d_{\text {max }}=(N-1) \cdot d_{\text {ant }}
$$

This length is twice the length of the classical DPCA system employing a single transmitter. The additional phase centers provide hence an increased number of azimuth samples along the synthetic aperture with the potential for improved ambiguity suppression. One may hence reduce either the PRF or the overall antenna length by a factor of two. Another opportunity is an enhanced detection and parameter estimation performance in a multi-baseline along-track interferometer and/or ground moving target indication (GMTI) system due to the increased length of the total along-track baseline. 


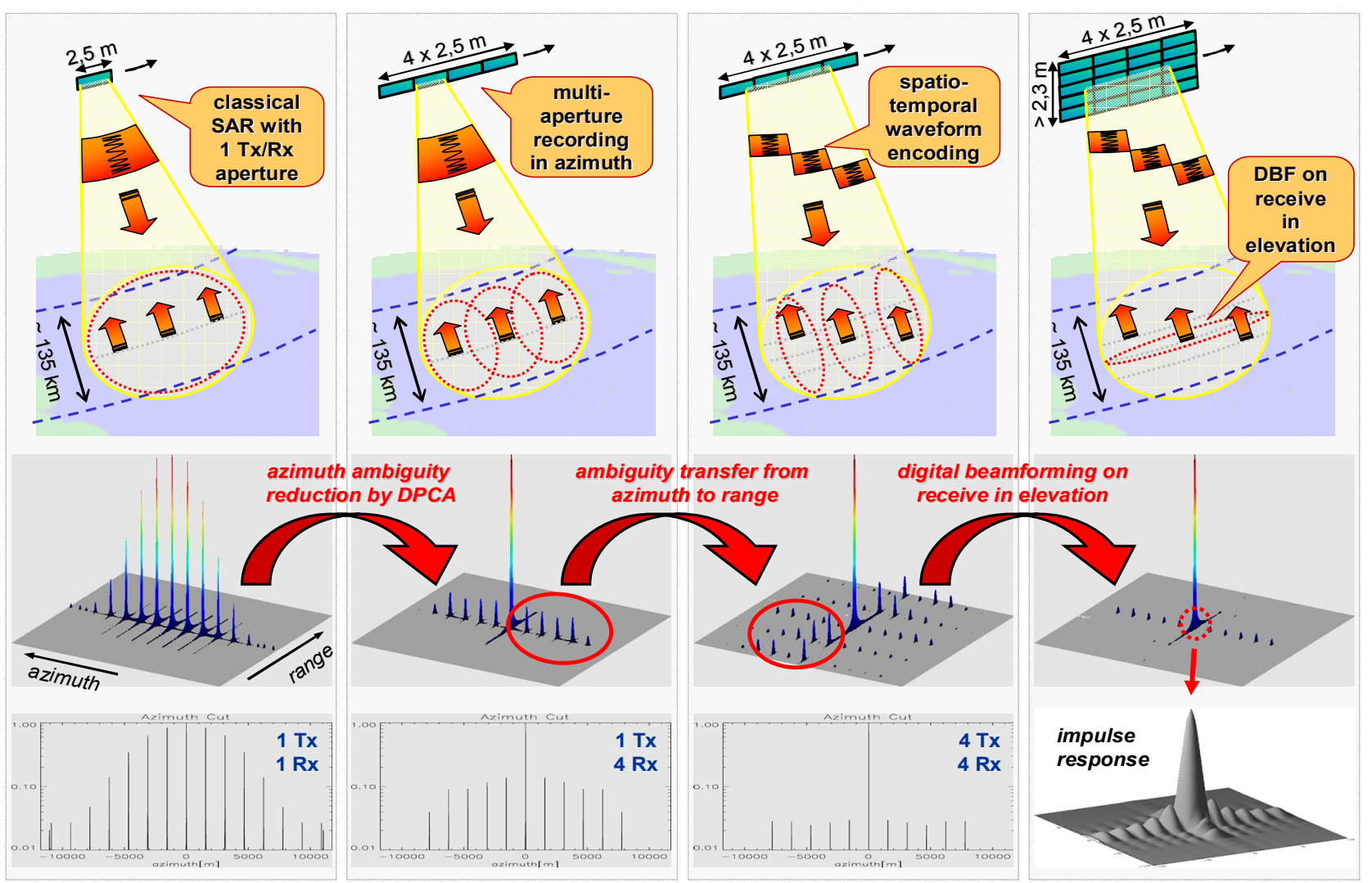

Figure 6. Transfer of ambiguous energy from azimuth to range by multidimensional waveform encoding for four transmit and four receive channels. Left: classical SAR with one transmitter and one receive aperture. Middle left: azimuth ambiguity suppression by a classical DPCA system with four independent receive apertures. Middle right: ambiguity transfer from azimuth to range by intra-pulse azimuth beamsteering with three sub-pulses. Right: range ambiguity suppression by digital beamforming on receive in elevation. The top row illustrates the aperture arrangement, the transmitted waveform and the spatial location of simultaneous returns from the ground, the middle row shows the processed SAR image from a single point-like scatterer in range and azimuth, and the bottom shows the magnitude of the azimuth ambiguities obtained from a slice through the processed SAR image. The shaded surface plot on the lower right is a 2-D zoom of the SAR impulse response.

On a first sight, one may believe that orthogonal Tx waveforms are also well suited to reduce azimuth ambiguities in a high-resolution wide-swath SAR imaging system. However, the mere use of simultaneously transmitted orthogonal waveforms will only disperse - but not suppress the ambiguous energy, thereby making this approach only suitable for the attenuation of ambiguous returns from point-like targets in specialized scenarios. A full suppression of ambiguous returns from distributed targets can, however, be obtained by combining the spatial transmit diversity in azimuth with digital beamforming on receive in elevation. For this, the orthogonal signals from the azimuth apertures are not transmitted simultaneously but in sequence by dividing the total Tx pulse again into multiple sub-pulses where the number of sub-pulses corresponds to the number of azimuth apertures. The scattered signals from the different sub-pulses will then -at each instant of time- arrive from different elevation angles and it becomes possible to separate the radar echoes from the different sub-pulses by digital beamforming on receive in elevation. This spatial filtering will hence suppress, and not only disperse, the ambiguous energy from distributed scatterers.

An alternative to the sequential transmission from multiple azimuth apertures is the formation of multiple narrow azimuth beams in the transmitter, thereby reducing the Doppler bandwidth in the receiver channels as schematically illustrated in Fig. 6 on the upper right. This solution has the advantage to use always all Tx antenna elements which alleviates the peak power requirements of the T/R modules to achieve a predefined signal-to-noise ratio. A sequence of chirp signals is then transmitted while switching between different azimuth beams from sub-pulse to sub-pulse. This specific illumination sequence results for each point on the ground in multiple and mutually delayed chirp signal returns. If we consider now a scatterer at a given range, one will at each instance of time only receive the scattered signal from one sub-pulse while the other sub-pulses lead to a superposition of the received signal with range ambiguous echoes from scatterers located at different ranges. These different ranges are in turn associated with different look angles in elevation. It is hence possible to suppress the ambiguous returns from different ranges by digital beamforming on receive in elevation which enables a clear and unambiguous separation of the received echoes from the different azimuth beams. The echoes from multiple azimuth beams are finally combined coherently to recover the full Doppler spectrum for high azimuth resolution. This combination is equivalent to a signal reconstruction from a multi-channel bandpass decomposition, where the individual bandpass signals correspond to narrow band azimuth spectra with different Doppler centroids. Fig. 6 illustrates the improved azimuth ambiguity suppression from multidimensional waveform encoding. A more detailed description and the corresponding processing algorithms can be found in [10]. 


\section{DISCUSSION}

The systematic combination of spatiotemporal radar waveform encoding on transmit with multi-aperture digital beamforming on receive is an innovative concept which enables new and very powerful SAR imaging modes for a wide range of remote sensing applications. Examples are an improved SAR system performance by increasing the number of effective phase centers, larger along-track baselines for along-track interferometry and moving object indication, and an efficient reduction of redundant information recorded by large receiver arrays. The opportunity to transfer ambiguous signal energy from azimuth to range via multi-beam switching during each transmitted radar pulse enables furthermore an efficient suppression of azimuth ambiguities by a spatiotemporal filtering of the recorded multi-aperture data in elevation. This paves the way for a new class of very powerful high-resolution widecoverage radar imaging systems.

Digital beamforming on transmit allows furthermore a flexible distribution of the RF signal energy on the ground. This enables not only a switching between different SAR modes like Spotlight, ScanSAR and HRWS stripmap, but it allows also for the simultaneous combination of multiple imaging modes in one and the same data acquisition. An example for such an interleaved operation is a spotlight imaging of an area of high interest in combination with a simultaneous wide swath SAR mapping for interferometric applications. This can be achieved by enhancing the multidimensional waveform encoding with additional subpulses that steer highly directive transmit beams to some specific areas on the ground. By this, one obtains a high Tx gain and a longer illumination time along the synthetic aperture, which will improve both the radiometric and the geometric resolution for the local areas of high interest. This is illustrated in Fig. 7 and such a hybrid mode is well suited to satisfy otherwise contradicting user requirements like the conflict between a continuous interferometric background mission and a high-resolution imaging request.

The data acquisition in such a hybrid system could even be made adaptive where more system resources are devoted automatically to areas of high interest and/or low SNR, thereby maximizing the overall information content for a given RF power budget. By this, a closed loop will be formed which connects the recorded data directly to the transmitted waveform by an appropriate real-time raw data evaluation of the scattered signals from the environment (cf. Fig. 3). As a simple opportunity one may consider the adaptive use of a longer sub-pulse and/or a higher Tx antenna gain for a spatially restricted area with lower backscatter, thereby enhancing the overall signal-to-noise ratio for a given amount of system resources. Such an adaptation can e.g. be performed in real-time via a multiple beamforming processor which evaluates the spatial power distribution of the scattered and recorded radar data. The output from this processor is then directly fed back to the waveform encoding system. Another opportunity is the use of longer azimuth illumination times and higher range bandwidths for selected regions with high contrast and/or fast changes. Such features could be indicators for areas of high interest, therefore calling also for a higher spatial and/or radiometric resolution. A potential application sce-

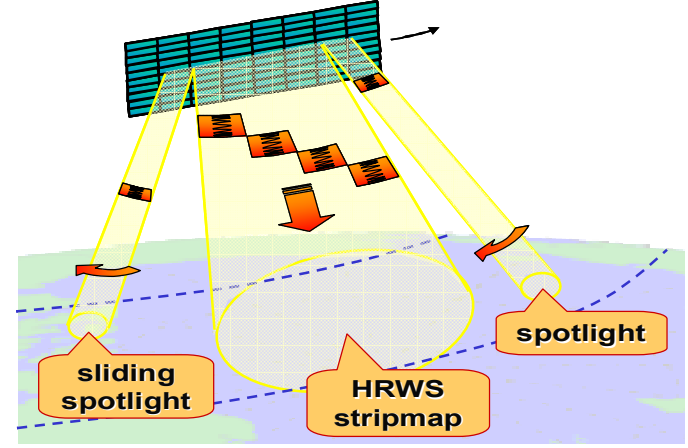

Figure 7. Hybrid SAR imaging modes.

nario is e.g. operational ship detection in the open sea which requires the frequent scanning of wide areas. In this scenario, the recorded multi-aperture signals may now be evaluated on board the satellite by a sub-optimum realtime MTI feature detector with low complexity. In case of some evidence for a moving object or ship in one area of the scene, one may illuminate that region with a highly directive sub-beam to improve both the spatial resolution and the MTI performance without loosing the general overview about the residual scene. Such a system can also be regarded as a first step towards a cognitive radar which directs its resources to areas of high interest in analogy to the selective attention mechanisms of the human visual system with its saccadic eye movements [11]. The rising interest in such systems is also well documented in the recent literature [12].

\section{REFERENCES}

[1] J. C. Curlander and R. N. McDonough, Synthetic Aperture Radar: Systems and Signal Processing. New York: Jon Wiley \& Sons, 1991.

[2] K. Tomiyasu, "Conceptual performance of a satellite borne, wide swath synthetic aperture radar," IEEE Transactions on Geoscience and Remote Sensing, vol. 19, pp. 108-116, 1981.

[3] W. Carrara, R. Goodman, and R. Majewski, Spotlight Synthetic Aperture Radar: Signal Processing Algorithms. Boston: Artech House, 1995.

[4] A. Currie and M. A. Brown, "Wide-swath SAR," IEE Proceedings F Radar and Signal Processing, vol. 139, pp. 122-135, 1992.

[5] G. D. Callaghan and I. D. Longstaff,"Wide-swath space-borne SAR using a quad-element array," IEE Proceedings on Radar, Sonar and Navigation, vol. 146, pp. 159-165, 1999.

[6] M. Suess, B. Grafmueller, and R. Zahn, "A novel high resolution, wide swath SAR system," in Proc. IEEE Geoscience and Remote Sensing Symposium, Sydney, Australia, pp. 1013-1015, 2001.

7] G. Krieger, N. Gebert, and A. Moreira, "Unambiguous SAR signal reconstruction from nonuniform displaced phase center sampling," IEEE Geoscience and Remote Sensing Letters, vol. 1, pp. 260-264, 2004.

[8] C. Fischer, C. Heer, G. Krieger, and R. Werninghaus, "A high resolution wide swath SAR," in Proc. European Conference on Synthetic Aperture Radar, Dresden, Germany, 2006.

[9] N. Gebert, G. Krieger, A. Moreira, "Digital Beamforming on Receive: Techniques and Optimization Strategies for High Performance SAR Imaging," submitted to IEEE Transactions on Geoscience and Remote Sensing, 2007.

[10] G. Krieger, N. Gebert, and A. Moreira, "Multidimensional Waveform Encoding: A New Digital Beamforming Technique for Synthetic Aperture Radar Remote Sensing," to appear in IEEE Transactions on Geoscience and Remote Sensing, 2007.

[11] G. Krieger, I. Rentschler, G. Hauske, K. Schill, and C. Zetzsche, "Object and Scene Analysis by Saccadic Eye-Movements: An Investigation with Higher-Order Statistics," Spatial Vision, vol. 13, pp. 201-214, 2000.

[12] F. Gini, "Knowledge-Based Systems for Adaptive Radar [Guest editorial]," IEEE Signal Processing Magazine, vol. 23, pp. 14-17, 2006. 